\title{
The E3 ubiquitin ligase Hace1 is required for early embryonic development in Xenopus laevis
}

\author{
Akira limura, Fuhito Yamazaki, Toshiyasu Suzuki, Tatsuya Endo, Eisuke Nishida and Morioh Kusakabe
}

\begin{abstract}
Background: HECT domain and ankyrin repeat containing E3 ubiquitin protein ligase 1 (HACE1) regulates a wide variety of cellular processes. It has been shown that one of the targets of HACE1 is the GTP-bound form of the small GTPase Rac1. However, the role of HACE1 in early development remains unknown.

Results: In situ hybridization revealed that Xenopus laevis hacel is specifically expressed in the ectoderm at the blastula and gastrula stages and in the epidermis, branchial arch, kidney, and central nervous system at the tailbud stage. Knockdown of hace1 in Xenopus laevis embryos via antisense morpholino oligonucleotides led to defects in body axis elongation, pigment formation, and eye formation at the tadpole stage. Experiments with Keller sandwich explants showed that hacel knockdown inhibited convergent extension, a morphogenetic movement known to be crucial for body axis elongation. In addition, time lapse imaging of whole embryos during the neurula stage indicated that hacel knockdown also delayed neural tube closure. The defects caused by hacel knockdown were partly rescued by knockdown of rac1. Moreover, embryos expressing a constitutively active form of Rac1 displayed phenotypes similar to those of embryos with hacel knocked down.
\end{abstract}

Conclusions: Our results suggest that Xenopus laevis hace1 plays an important role in early embryonic development, possibly via regulation of Rac1 activity.

Keywords: HACE1, Early development, Xenopus laevis

\section{Background}

HECT domain and ankyrin repeat containing E3 ubiquitin protein ligase 1 (HACE1) was identified as a gene located in an affected region of chromosome $6 \mathrm{q} 21$ in human Wilms' tumor [1]. It has been shown that $H A C E 1$ expression is reduced in multiple human tumors, and forced expression of HACE1 in human tumor cell lines inhibits cell growth in vitro and in vivo [2]. Moreover, Hace ${ }^{-1-}$ mice develop spontaneous lateonset cancer [2]. Therefore, HACE1 is considered to be a tumor suppressor gene. HACE1 protein is a HECT family E3 ligase and is known to regulate multiple cellular processes. The most investigated target of HACE1 is Rac1. HACE1 specifically recognizes GTP-bound Rac1,

\footnotetext{
* Correspondence: tsuzuki.m07@lif.kyoto-u.ac.jp

Department of Cell and Developmental Biology, Graduate School of Biostudies, Kyoto University, Sakyo-ku, Kyoto 606-8502, Japan

an active form of Rac1, and promotes its degradation through the ubiquitin-proteasome pathway [3]. Rac1 degradation by HACE1 is important in regulating cell migration and reactive oxygen species (ROS) production $[4,5]$. In addition, Rab GTPases are binding partners of HACE1 [6]. HACE1 regulates Golgi membrane dynamics during the cell cycle by interacting with Rab proteins [6]. One Rab GTPase Rab11a is activated by HACE1mediated ubiquitination and subsequently promotes recycling of the $\beta_{2}$-adrenergic receptor [7]. In addition, HACE1 has protective actions against oxidative stress in the brain and hemodynamic stress in the heart $[8,9]$. Thus, these findings have demonstrated HACE1 functions in diverse cellular processes. However, the roles of HACE1 during vertebrate embryonic development have not been previously reported in any model organism.

Here, we show that Xenopus laevis hace1 is mainly expressed in the ectoderm and plays an important role in embryonic development. Hace1-depleted Xenopus 
laevis embryos displayed defects in multiple developmental processes. Analysis with Keller sandwich explants revealed that Xenopus laevis hace1 is essential for convergent extension.

\section{Results}

Xenopus laevis hace 1 is mainly expressed in the ectoderm during early embryogenesis

To investigate a role of HACE1 in early embryonic development, we first cloned a Xenopus laevis HACE1 homolog (accession number AB894419) with a 2739 bp open reading frame. Compared with the reported sequence of Xenopus laevis hace1 in GenBank (accession number NM_001093608), the Xenopus laevis hace1 sequence that we obtained had five silent point mutations and a 96 bp deletion. These silent mutations may be single nucleotide polymorphisms. The $96 \mathrm{bp}$ deletion may be caused by alternative splicing, because the human HACE1 gene deposited in GenBank (accession number NM_020771) has a similar deletion. We thus concluded that the obtained Хenopus laevis hace1 clone is a splicing variant. Human HACE1 protein shares $90 \%$ and $87 \%$ amino acid identity with our Xenopus laevis Hace1 protein and the previously reported protein, respectively. Hence, we used our clone in this study.

Whole-mount in situ hybridization was performed to determine the expression pattern of Xenopus laevis hace1. Maternal transcripts of hace1 were localized to the animal region (Fig. 1a). The expression was high in the presumptive ectoderm at the gastrula stage (Fig. 1b). At the neurula stage, hace1 was weakly expressed in the dorsal ectoderm (Fig. 1c). At the early tailbud stage, hace1 showed a spotty expression pattern in the epidermis and a uniform expression pattern in the neural tube (Fig. 1d). At the late tailbud stage, hace1 was expressed in the brain, eye, neural tube, branchial arch, ear vesicle, and kidney (Fig. 1e).

\section{Knockdown of hace1 causes shortening of the body axis and inhibition of eye and pigment formation}

We next performed knockdown experiments in developing Xenopus laevis embryos by using an antisense morpholino oligonucleotide against hace1 (HACE1 MO). We designed HACE1 MO to target the sequence containing the translation initiation AUG of hace1 mRNA. To evaluate the specificity and efficiency of HACE1 MO, we performed immunoblotting analysis. HACE1 MO blocked the translation of C-terminally Myc-tagged hace1 (hace1-myc) mRNA containing the HACE1 MO target sequence (Fig. 2a, left two lanes). We then injected HACE1 MO into the animal region of dorsal or ventral blastomeres at the 4-cell stage and observed the embryos at the tadpole stage (stage 4041). Embryos that were dorsally injected with HACE1 MO displayed a number of defects, including a shortened body axis, a reduction of pigments, and a reduction of eye size (Fig. 2b, middle panel), whereas embryos that were ventrally injected with HACE1 MO exhibited no significant phenotype (data not shown). According to the extent of each defect, we classified embryos into three groups: severe, mild, and normal (Fig. 2c, d). Coinjection of HACE1 MO and N-terminally Myc-tagged hace1 (myc-hace1) mRNA, which was MO-resistant because of the insertion of two Myc tags near the MO recognition site (Fig. 2a, middle and second right lanes), resulted in milder defects than the injection of HACE1 MO alone (Fig. 2b-f), indicating that the phenotypes observed in the HACE1 MO-injected embryos were primarily caused by Hacel depletion. Thus, these results suggest that hace1 has an important role in early Xenopus laevis embryonic development.

\section{Hace 1 is required for convergent extension}

In early Xenopus laevis development, dorsal mesodermal and ectodermal cells converge toward the midline and mediolaterally intercalate, and this process, called convergent extension, leads to elongation of the anterior-posterior body axis [10]. Therefore, it can be hypothesized that the shortened body axis observed in hace1 knockdown embryos is caused by aberrant convergent extension. To test whether hace1 regulates convergent extension, we used Keller sandwich explants, in which convergent extension can be reproduced in culture [11]. Explants from embryos injected with control MO (Fig. 3a, left panel) elongated normally, whereas those from embryos injected with HACE1 MO displayed little elongation (Fig. 3a, middle panel). The inhibition of elongation by HACE1 MO was rescued by coinjection of myc-hace1 mRNA (Fig. 3a, right panel). To evaluate the elongation of explants, we measured the length-to-width ratio of explants. The length-to-width ratio was significantly reduced by HACE1 $\mathrm{MO}$, and this reduction was significantly rescued by coinjection of myc-hace1 mRNA (Fig. 3b). These results suggest that Hace1 regulates convergent extension.

\section{Hace1 depletion causes a delay in neural tube closure}

Because HACE1 MO inhibited convergent extension in Keller sandwich explants, we examined the effect of HACE1 MO on gastrulation and neural tube closure, which are two major morphological events involving convergent extension, in whole embryos. An injection of $30 \mathrm{ng}$ of HACE1 MO did not lead to an apparent defect in gastrulation (data not shown), but it led to a delay in neural tube closure at the neurula stage (Fig. 4a). To examine this phenotype in detail, we performed timelapse imaging of neurulating embryos. At the 4-cell stage, HACE1 MO was injected into the right dorsal blastomere, and control MO was injected into the left dorsal blastomere (Fig. 4b-d, indicated as HACE1 MO). As a control, 
a

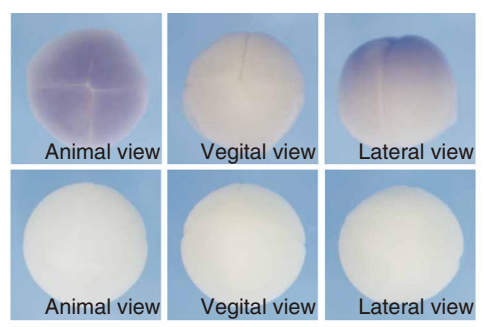

C
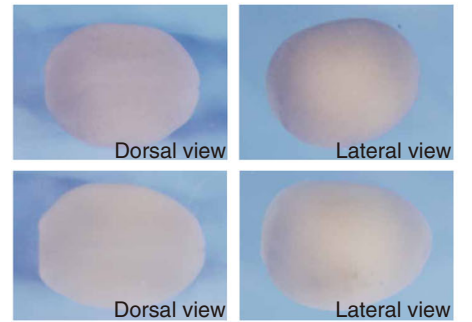

d
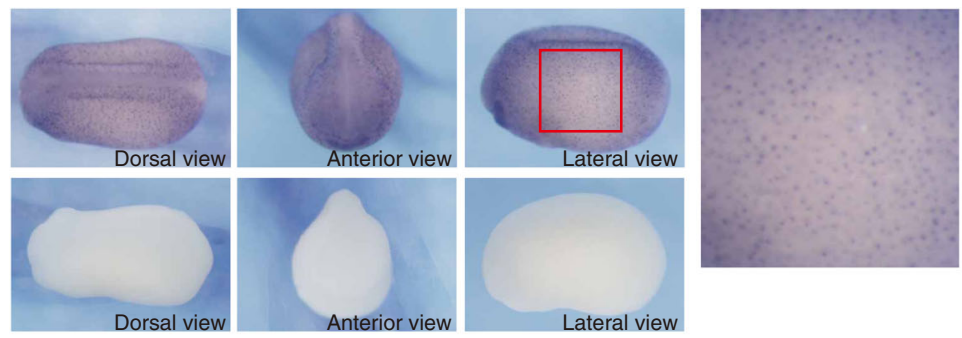

e
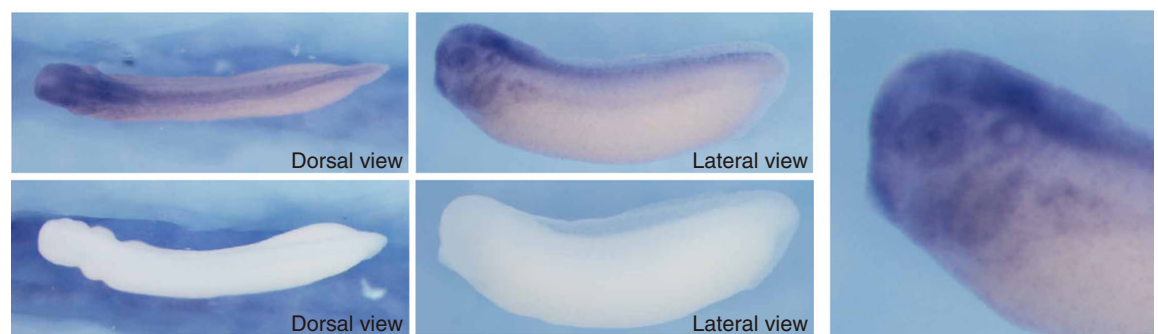

Fig. 1 Spatial expression of Xenopus laevis hacel in early development. Expression of hacel during Xenopus laevis development was analyzed by whole-mount in situ hybridization using an antisense probe (upper panels) and sense probe as a control (lower panels). a, b At stage 4 (a) and 11 (b), hacel was expressed throughout the presumptive ectoderm. c At stage 15, hacel expression was weakly detected at the dorsal ectoderm. d At stage 23, hacel was expressed in the neural tissue and also showed spotty expression in the epidermis. The rightmost panel shows a magnification of the red boxed region. e hacel was expressed in the brain, eye, neural tube, branchial arch, ear vesicle, and kidney at stage 31. The right panel shows a magnified view of the embryo. Animal is to the top in lateral views $(\mathbf{a}, \mathbf{b})$. Dorsal is to the top in lateral or anterior views $(\mathbf{c}, \mathbf{d}, \mathbf{e})$. Anterior is to the left in dorsal or lateral views $(\mathbf{c}, \mathbf{d}, \mathbf{e})$

uninjected embryos were also observed (Fig. 4b-d, uninjected). Embryos at stage 13 were placed on a culture dish, and images were collected every 5 minutes until neural tube closure was completed (Fig. $4 \mathrm{~b}-\mathrm{d}$ and see also Additional file 1: Movie S1 and Additional file 2: Movie S2; uninjected embryos and $\mathrm{MO}$-injected embryos, respectively). The neural plate was likely to be formed in the HACE1 MO-injected right side (Fig. 4b, 0 min). However, the elevation of the neural fold was much less apparent in the HACE1 MO-injected side than in the control MOinjected side (Fig. 4b, the middle panel at $60 \mathrm{~min}$ ). Although the neural fold became visible at 30 to $60 \mathrm{~min}$ in the HACE1 MO-injected right side, the ridge of the neural fold in the right side was much less prominent than that in the control MO-injected left side (Fig. 4b, $120 \mathrm{~min})$. We measured the maximum distance between the dorsal midline and the inner edge of the neural folds. In uninjected embryos, the distance in 


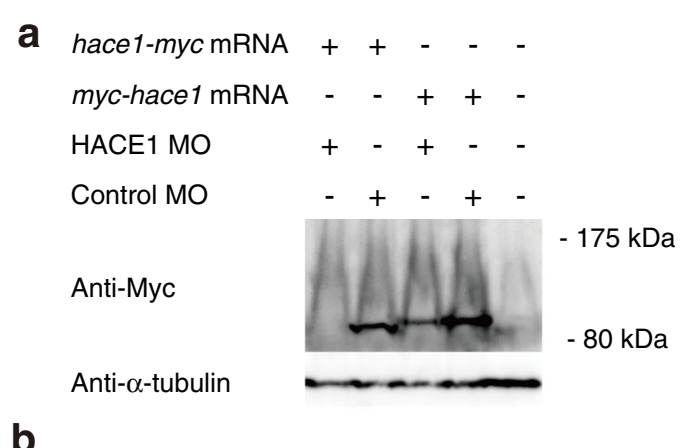

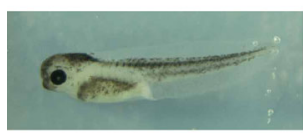

Uninjected

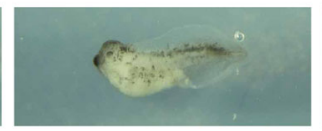

HACE1 MO

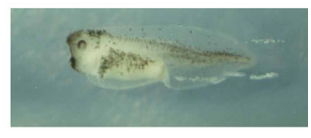

HACE1 MO + myc-hace1 mRNA

C

d

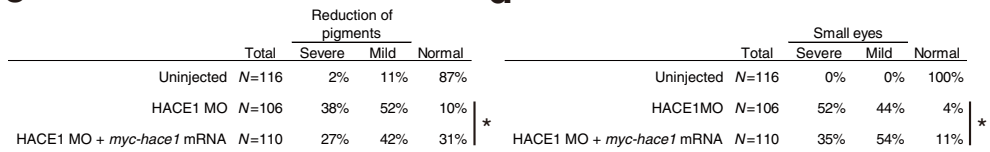

e

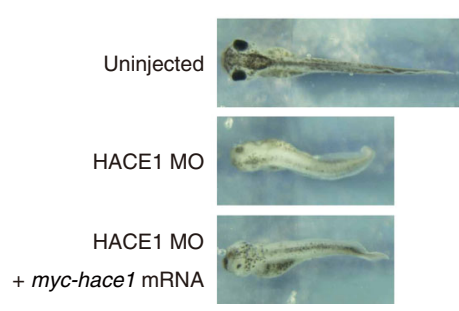

f

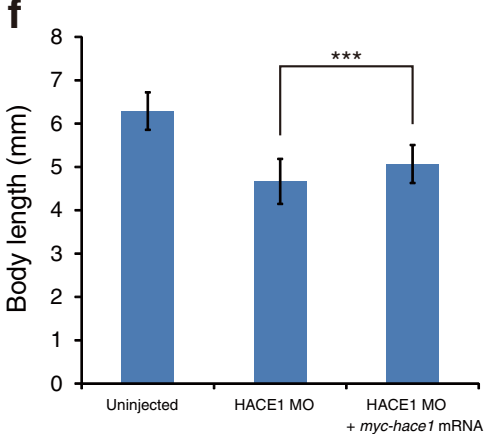

Fig. 2 Dorsal depletion of hacel leads to multiple developmental defects. a Immunoblot analysis shows that HACE1 MO inhibited translation of hace1-myc mRNA but not that of myc-hacel mRNA used for rescue experiments. Xenopus laevis embryos were injected with the indicated sets of MOs (30 ng) and mRNAs (1.2 ng). a-tubulin was a loading control. b Embryos were injected with $30 \mathrm{ng}$ of HACE1 MO alone (middle panel) or $30 \mathrm{ng}$ of HACE1 MO plus $1.2 \mathrm{ng}$ of myc-hace1 mRNA (right panel) into the animal region of two dorsal blastomeres at the four-cell stage. $\mathbf{c}$, $\mathbf{d}$ Embryos were classified into severe, mild, and normal groups on the basis of the extent of each defect. * $P<0.05$ (with Bonferroni correction), Mann-Whitney $U$-test. c $N$, number of total right and left sides of embryos we evaluated. The right side and left side of each embryo were separately evaluated. $\mathbf{d} N$, number of total eyes we evaluated. The right eye and left eye of each embryo were separately evaluated. e, $\mathbf{f}$ The body length of embryos at stage 40-41 was quantified (Uninjected, $n=59$; HACE1 MO, $n=53$; HACE1 MO + myc-hace1 mRNA, $n=55$ ). Values are expressed as the mean \pm s.d. ***P 0.001 (with Bonferroni correction), unpaired $t$-test

the right side was roughly equal to that in the left side (Fig. 4c, d). However, the distance in the HACE1 $\mathrm{MO}$-injected right side was markedly longer than that in the control MO-injected left side (Fig. 4c, d), thus indicating that knockdown of hace1 led to a significant delay in neural tube closure. These results suggest that hace1 is required for normal neural tube closure.

\section{Phenotypes of hace1 morphants are specific for loss of hace 1}

Knockdown by antisense morpholino oligonucleotides may be accompanied by off-target effects [12]. Although we performed rescue experiments (Fig. 2b-f), we further evaluated the specificity of hace1 knockdown. We designed HACE1 MO2 as a second non-overlapping morpholino oligo for hace1. Whereas HACE1 MO targets a region from -1 to +24 from the translational start site, HACE1 MO2 targets a region from -79 to -55 from the translational start site. Embryos dorsally injected with HACE1 MO2 displayed a shortened body axis, a reduction of pigments, and a reduction of eye size (Fig. 5a-e). We next examined the effect of HACE1 MO2 at an early developmental stage. Similarly to the results from the injection of HACE1 MO, the injection of HACE1 MO2 did not lead to an apparent defect in gastrulation (data 


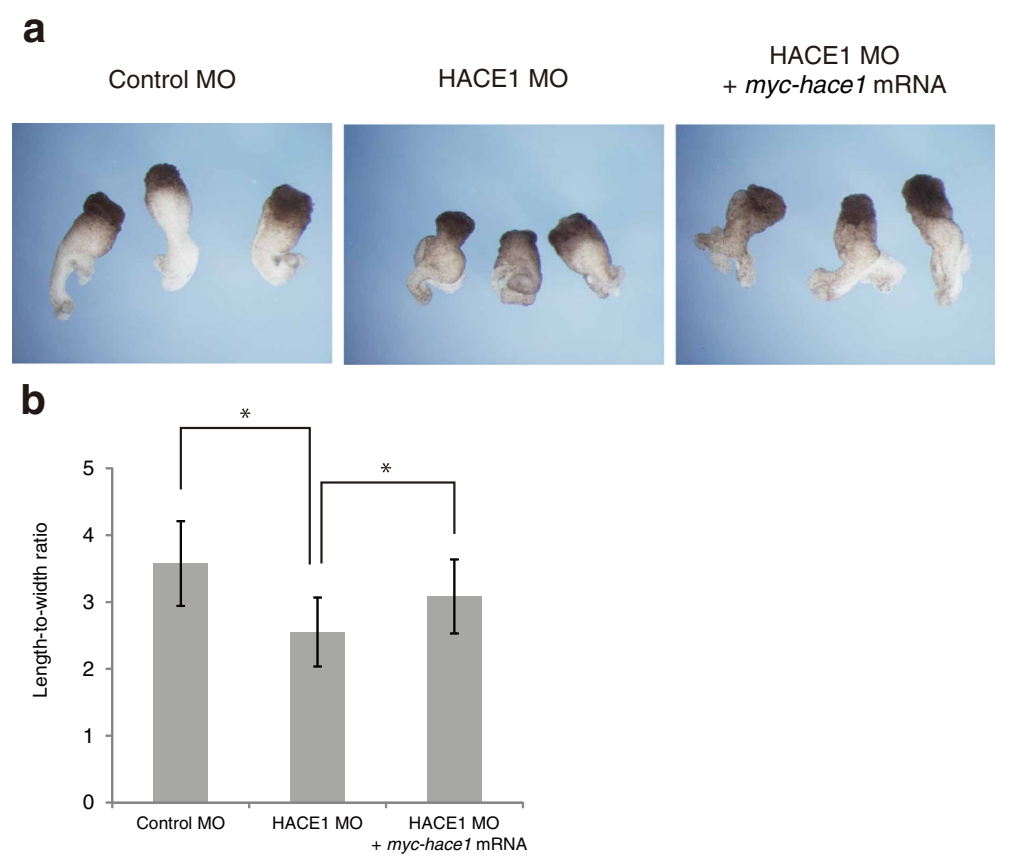

Fig. 3 hacel knockdown inhibits convergent extension. a Keller sandwich explants were prepared from embryos injected with 30 ng of control MO or HACE1 MO into dorsal blastomeres at the four-cell stage. HACE1 MO (middle panel, $n=18$ ), but not control MO (left panel, $n=18$ ), caused a defect in elongation of explants. The defect in elongation caused by HACE1 MO was partly rescued by coinjection of 1.2 ng of myc-hace 1 mRNA (right panel, $n=18$ ). $\mathbf{b}$ The length-to-width ratio of explants was quantified. Values are expressed as the mean $\pm s$.d. ${ }^{*} P<0.05$, unpaired $t$-test

not shown), but it did lead to a delay in neural tube closure at the neurula stage (Fig. 5f). Thus, knockdown of hace 1 by HACE1 MO2 led to essentially the same phenotype as that by HACE1 MO. These results further confirmed the specificity of morpholino-mediated knockdown of hace1.

\section{Developmental defects in hace1 morphants are largely caused by an excess of active Rac1}

HACE1 ubiquitinates GTP-bound RAC1 and causes its degradation [3]. Thus, it is possible that the phenotype of hace 1 morphants is caused by an increase in active Rac1. To investigate the relationship between hace1 and rac, we first attempted to analyze the expression pattern of the rac family genes: $r a c 1, r a c 2$ and $r a c 3$. It has previously been shown that Xenopus laevis rac1 is expressed in neural tissues at the neurula and tailbud stages [13]. Our qRT-PCR analysis showed that rac1 is expressed throughout early development, and rac2 expression starts from the early tailbud stage (Fig. 6a). The fulllength coding sequence of Xenopus laevis rac3 has not been deposited in GenBank, and we were unable to find Xenopus laevis EST sequences corresponding to rac3. In addition, we were unable to amplify any DNA fragments from Xenopus laevis embryos in our RT-PCR experiments using primers that were designed on the basis of the rac3 sequence from the closely related species Xenopus (Silurana) tropicalis, thus suggesting that rac3 may be absent or weakly expressed in Xenopus laevis embryos. These results indicate that rac1 is predominantly expressed among rac family members at the neurula stage. Thus, it is possible that hace1 targets rac1 in Xenopus laevis neurulation.

We next asked whether the defects observed in hace1 morphants could be rescued by reducing Rac1 activity. We used Rac1 MO to reduce the total Rac1 protein level. Immunoblotting analysis confirmed that Rac1 MO reduced the protein level of endogenous Rac1 by more than half (Fig. 6b). We injected HACE1 MO with control MO or Rac1 MO into embryos and fixed them at the tadpole stage (stage 40-41). Embryos coinjected with HACE1 MO and Rac1 MO displayed milder defects in eyes and pigments than did embryos coinjected with HACE1 MO and control MO, although these double MO-injected embryos displayed more severe phenotypes than embryos injected with HACE1 MO alone, possibly because of overdosing of MOs (Fig. 6c-e). In addition, embryos coinjected with HACE1 MO and Rac1 MO had a longer body length than did embryos coinjected with HACE1 MO and control MO (Fig. 6f, g). These results suggest that the defects in hace1 morphants are at least partly caused by an excess of active Rac1. 
a
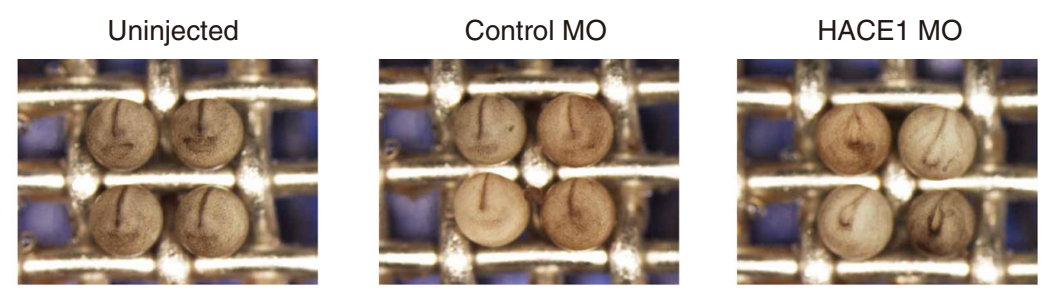

b

b Uninjected
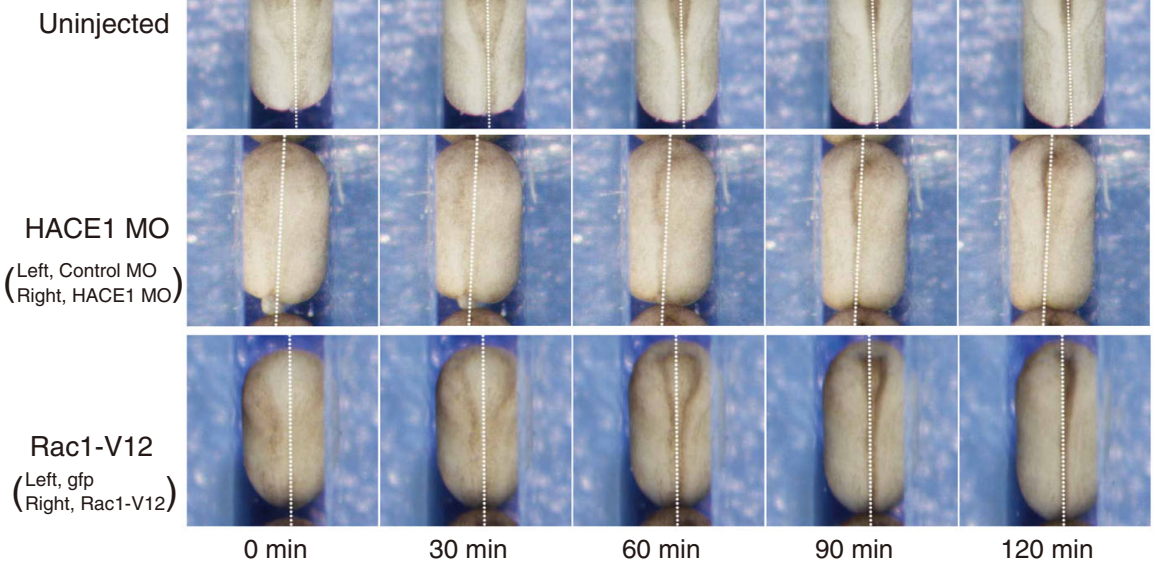

C

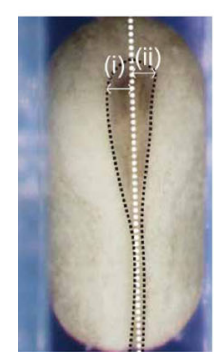

Uninjected

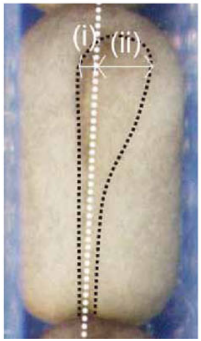

HACE1 MO

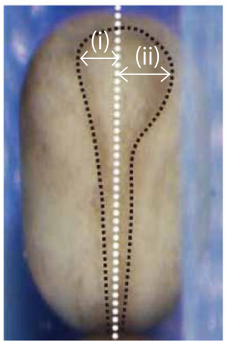

Rac1-V12 d

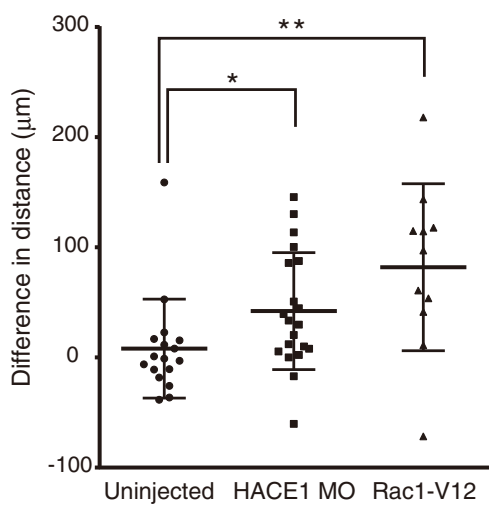

Fig. 4 Knockdown of hacel and overexpression of rac1-V12 cause a delay in neural tube closure. a Embryos were injected with the indicated MOs (30 ng) into the animal region of two dorsal blastomeres at the four-cell stage and the images were collected at stage 20. Anterior views with dorsal to the top. Representative results from two independent experiments are shown. b HACE1 MO (15 ng) was injected into the animal region of the right dorsal blastomere, and control MO (15 ng) was subsequently injected into that of the left dorsal blastomere. rac1-V12 (constitutively active Rac1) mRNA (10 pg) and gfp mRNA (90 pg) were injected into the animal region of the right dorsal blastomere, and then $g f p$ mRNA alone was injected into the animal region of the left dorsal blastomere. Embryos at stage 13 were placed on a culture dish, and images were collected every 5 min until embryos completed neural tube closure. Still frames from a time-lapse movie show embryos from stage 14 (0 min) to stage $18(120 \mathrm{~min}$ ). Dorsal views with anterior to the top. Dotted lines indicate the midline. Transient posterior protrusion (e.g., middle left) was sometimes observed because embryos were firmly embedded in the agarose groove and thus were constantly subjected to the lateralto-medial force. c A magnified view of embryos in b $(60 \mathrm{~min})$. Black dotted lines indicate the inner edges of neural folds. $\mathbf{d}$ The maximum distance between the dorsal midline and the left (i) or right (ii) inner edge of the neural fold was measured (shown as white double-headed arrows in c). The differences ((ii) minus (i)) were then calculated and plotted (Uninjected, $n=17$; HACE1 MO, $n=20$; Rac1-V12, n=11). Long central bar marks mean and upper and lower bars mark s.d. ${ }^{*} P<0.05,{ }^{*} P<0.01$, Mann-Whitney U-test

To further test this possibility, we expressed rac1-V12, a constitutively active mutant of $\mathrm{racl}$, in the presumptive neural plate to examine the effect of excessive active
Rac1 on neurulation. Nearly half of the embryos injected with rac1-V12 mRNA displayed a phenotype similar to that of embryos injected with HACE1 MO, such as a 
a

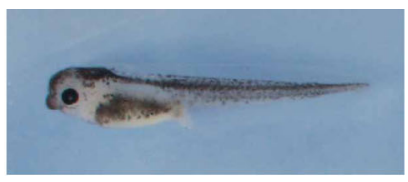

Control MO

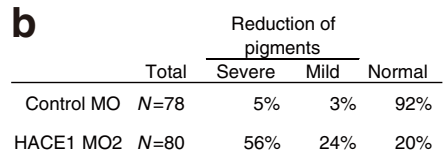

d
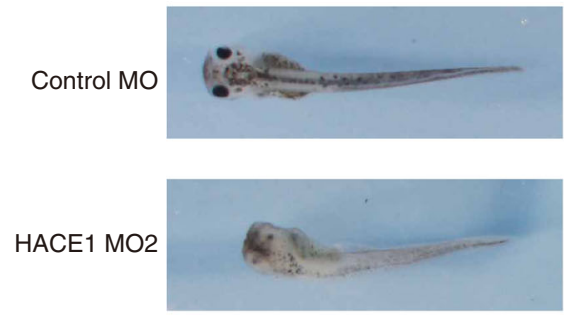

f

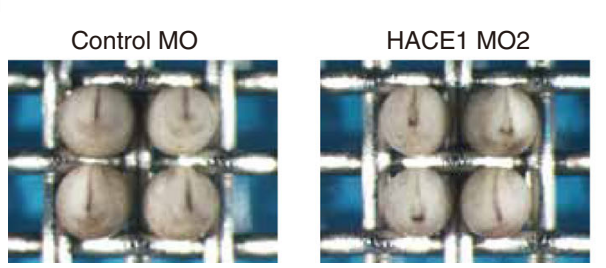

C

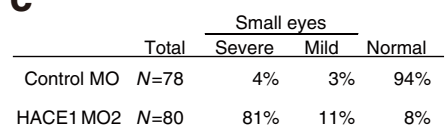

e

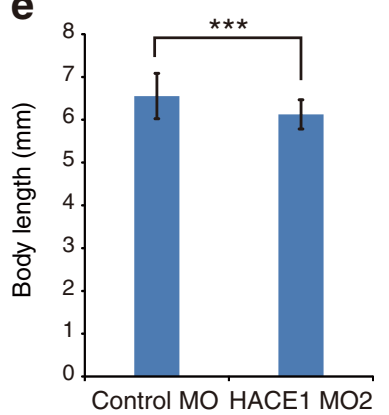

Fig. 5 Knockdown of hace1 by HACE1 MO2 leads to a phenotype similar to that caused by HACE1 MO treatment. a Embryos were injected with $50 \mathrm{ng}$ of Control MO (left panel) or $50 \mathrm{ng}$ of HACE1 MO2 (right panel) into dorsal blastomeres at the four-cell stage and fixed at stage 38/39. Representative results from two independent experiments are shown (Control $\mathrm{MO}, n=39 ; \mathrm{HACE} 1 \mathrm{MO}, n=40)(\mathbf{b}, \mathbf{c})$ Embryos were classified into severe, mild, and normal groups on the basis of the extent of each defect. $\mathbf{b} \mathbf{N}$, number of total right and left sides of embryos we evaluated. The right side and left side of each embryo were separately evaluated. c $N$, number of total eyes we evaluated. The right eye and left eye of each embryo were separately evaluated. d, e The body length of embryos at stage $38 / 39$ was quantified (Control MO, $n=39$; HACE1 MO2, $n=40$ ). Values are expressed as the mean $\pm s . d$. ${ }^{* * *} p<0.001$, unpaired $t$-test. $\mathbf{f}$ Embryos were injected with $50 \mathrm{ng}$ of Control MO (left panel) or $50 \mathrm{ng}$ of HACE1 MO2 (right panel) into dorsal blastomeres at the four-cell stage and the images were collected at stage 20 (Control MO, $n=40$; HACE1 MO2, $n=40$ ). Anterior views with dorsal to the top

shortened body axis (Fig. 6h, compare with Fig. 2b, middle), thus supporting our idea that developmental defects in hace1 knockdown embryos are at least partly due to excessive active Rac1. Because it has previously been reported that exogenous expression of rac1-V12 in the mesoderm ( 1 or $2 \mathrm{ng}$ of rac1-V12 mRNA into the marginal zone of embryos) results in gastrulation defects [14], we carefully observed the effect of rac1-V12 on gastrulation and showed that injection of rac1-V12 mRNA in our experimental condition (30 pg of mRNA was injected into the animal region of two dorsal blastomeres at 4-cell stage) did not affect gastrulation (data not shown).

\section{Excess of active Rac1 causes a delay in neural tube closure}

To further characterize the phenotype of the embryos injected with rac1-V12 mRNA, we performed time-lapse imaging and quantified the delay in neural tube closure. Embryos in which rac1-V12 mRNA was injected into the right side showed a significant delay in neural tube closure in the injected side, as compared with uninjected embryos (Fig. 4b-d and see also Additional file 1: Movie S1 and Additional file 3: Movie S3; uninjected embryos and rac1V12 mRNA-injected embryos, respectively). These results support our hypothesis that hace1 regulates Xenopus laevis neural tube closure through Rac1 degradation. 

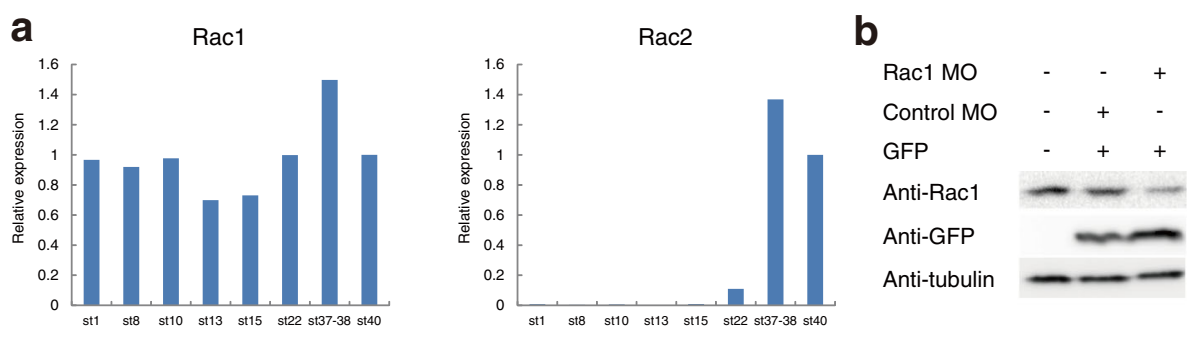

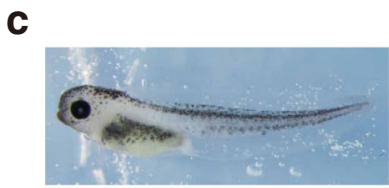

Uninjected

d

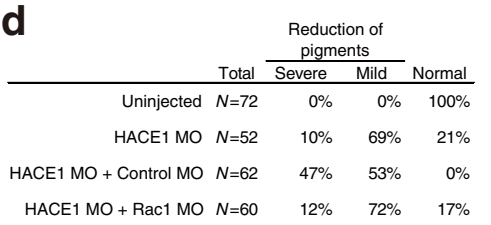

f

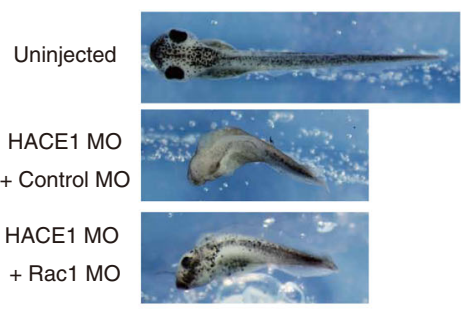

h

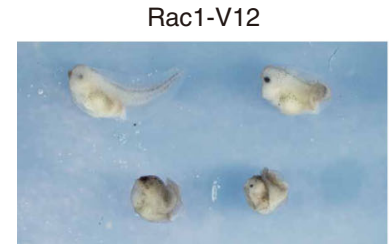

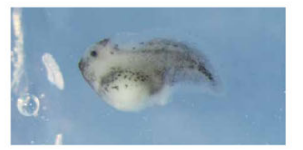

HACE1 MO + Control MO

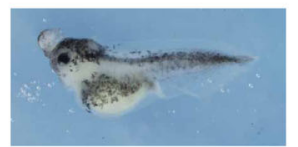

HACE1 MO + Rac1 MO

e
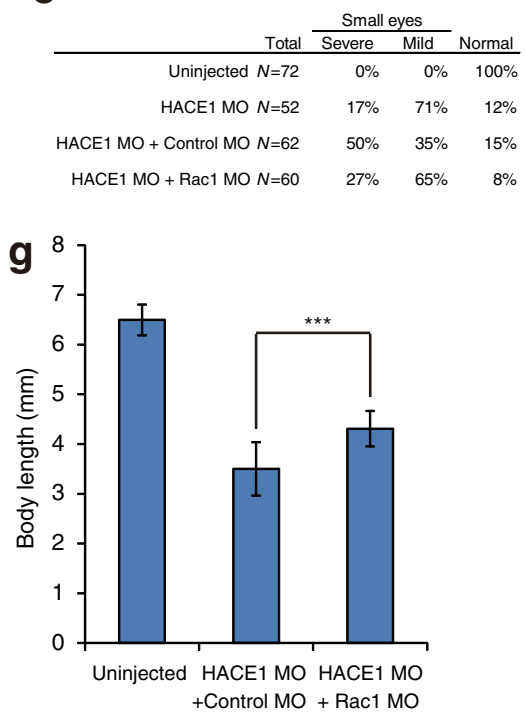

Fig. 6 Defects in hacel morphants are partly rescued by coinjection of Rac1 MO. a Temporal expression patterns of Xenopus laevis rac1 and rac2. The expression levels of racl and rac2 were analyzed by qRT-PCR. Expression levels were normalized to those of Xenopus laevis ornithine decarboxylase 1 (odc1). b Immunoblot analysis shows that Rac1 MO inhibited translation of endogenous Rac1. Xenopus laevis embryos were injected with the indicated MOs (70 ng in total) and gfp mRNA (0.2 ng). a-tubulin was a loading control. c Coinjection of Rac1 MO (50 ng) and HACE1 MO (30 ng) led to milder developmental defects than did coinjection of control MO (50 ng) and HACE1 MO (30 ng). Anterior is to the left, and dorsal is to the top. $\mathbf{d}$, e Embryos were classified into severe, mild, and normal groups on the basis of the extent of each defect. $\mathbf{f}$, $\mathbf{g}$ The body length of embryos at stage 40-41 was quantified. Dorsal views of embryos are shown with anterior to the left. Values are expressed as the mean \pm s.d. ${ }^{* *} P<0.001$, unpaired t-test $\mathbf{h}$ Embryos injected with 30 pg of Rac1-V12 (constitutively active Rac1) mRNA and 170 pg of gfp mRNA displayed phenotypes similar to those of embryos injected with HACE1 MO $(n=38)$. Anterior is to the left, and dorsal is to the top

\section{hace1 has a role in regulating differentiation to neural ectoderm}

Although the results shown above suggest that convergent extension is a major process affected by hacel depletion, it is possible that hace1 regulates cell fate specification, such as differentiation into the epidermal ectoderm, neural ectoderm, and mesoderm. To test this possibility, we analyzed the expression of marker genes of cell fate specification in hace1 depleted embryos. HACE1 MO was injected into the right side, and control MO was injected into the left side; the expression of marker genes was detected by in situ hybridization. Whereas the expression of the epidermal ectoderm marker epidermal keratin and the mesoderm marker 
Xbra were unaffected on the HACE1 MO-injected side (Fig. 7a, c), hace1 depletion resulted in a moderate or slight decrease in the expression of the neural ectoderm marker sox 2 at stage 12 (late gastrula) or stage 15 (early neurula), respectively (Fig. 7b). Additionally, the distribution pattern of all of these marker genes was essentially unaffected in HACE1 MO-injected side. According to the extent of reduction, we classified embryos into four groups: severe, moderate, slight, and normal (Fig. 7a-c). These results suggested that hace1 is likely to be involved

\section{a}

\begin{tabular}{llcccc} 
& & \multicolumn{3}{c}{ Reduction of expression } & \\
\cline { 3 - 5 } & Total & Severe & Moderate & Slight & Normal \\
\hline Stage 12 & $N=12$ & $0 \%$ & $8 \%$ & $17 \%$ & $75 \%$ \\
Stage 15 & $N=11$ & $0 \%$ & $0 \%$ & $36 \%$ & $64 \%$
\end{tabular}
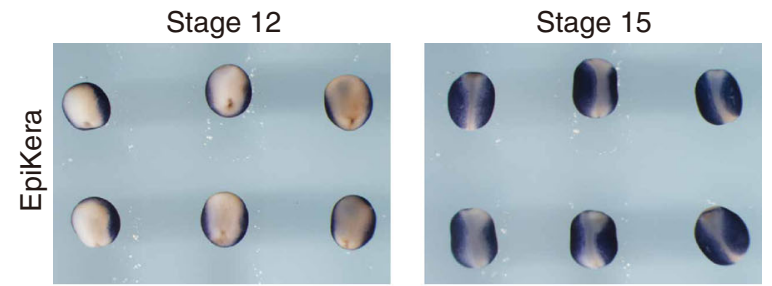

\section{b}

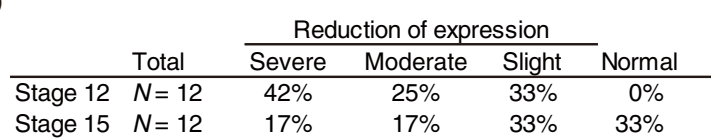

Stage 12
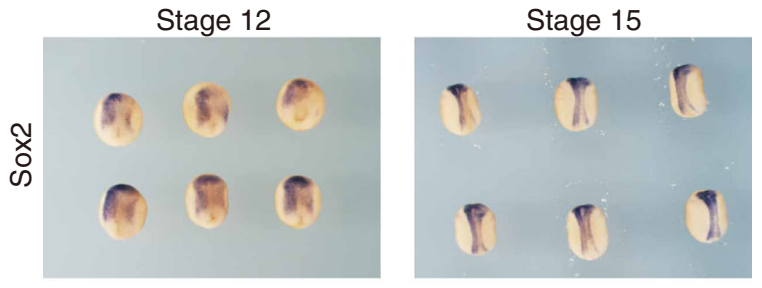

C

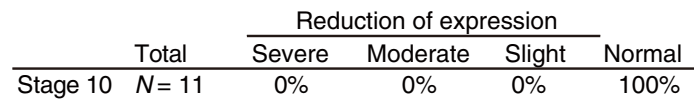

Stage $12 N=12=0 \% \quad 0 \%=10 \%$

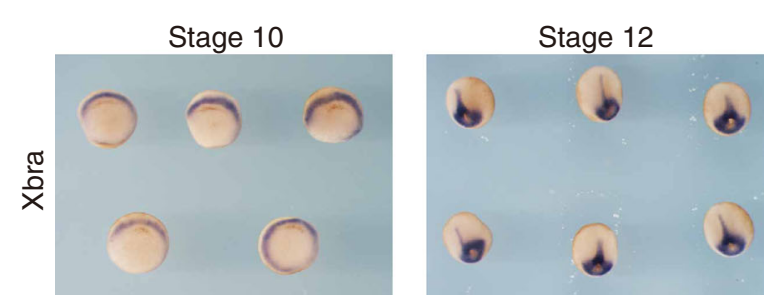

Fig. 7 hace 1 has a role in regulating differentiation into neural ectoderm. a-c HACE1 MO (15 ng) was injected into the animal region of the right dorsal blastomere, and control MO (15 ng) was injected into that of the left dorsal blastomere at the 4-cell stage. Embryos were fixed at the stages indicated, and expression of epidermal keratin, an epidermal ectoderm marker (a), sox2, a neural ectoderm marker (b), and Xbra, a mesodermal marker (c), were analyzed by in situ hybridization. Representative images from two independent experiments are shown (lower panels) in differentiation to the neural ectoderm. Although it is difficult to determine whether this level of decrease in Sox 2 affects the subsequent neural tube closure event, hace1 might regulate neural tube closure through controlling differentiation to the neural ectoderm.

\section{Discussion}

In this study, we investigated the role of the E3 ubiquitin ligase Hace1 in early embryonic development. We showed that Xenopus laevis hace1 is expressed in neural tissues and the kidney. High-throughput in situ hybridization in mouse embryos has revealed that Hace1 is expressed in neural tissues in mice at embryonic day 11.5 (E11.5), E15.5, and postnatal day 7 [15]. In humans, HACE1 is expressed in adult tissues including the heart, brain, and kidney [1]. These data suggest that HACE1 expression in neural tissues is common in vertebrates.

Hace1 depletion in Xenopus laevis embryos led to a shortened body axis. The process of body axis elongation is known to involve convergent extension [10, 16, 17]. Our analysis with Keller sandwich explants showed that knockdown of hace1 inhibited convergent extension. These results suggest that Xenopus laevis hace1 may control body axis elongation through regulation of convergent extension. Convergent extension occurs in both dorsal mesodermal and posterior neural tissues. Whereas convergent extension in the dorsal mesoderm mainly regulates gastrulation, convergent extension in the neural ectoderm mainly regulates neurulation. It has been shown using transplantation experiments that inhibition of convergent extension in only the neural ectoderm leads to a shortened body axis [18]. Because knockdown of hace1 did not affect gastrulation, inhibition of convergent extension in the neural ectoderm by hace1 depletion might lead to a shortened body axis.

Notably, Hace ${ }^{-/-}$mice do not display any developmental defects [2], whereas knockdown of hace1 in Xenopus laevis led to severe developmental defects. This discrepancy might be explained by the possibility that other proteins with roles redundant with that of HACE1 may compensate for HACE1 function during embryonic development of $\mathrm{HaCe}^{-/-}$mice. For instance, the activity of Rac1, one of the targets of HACE1, is negatively regulated by GAP and other E3 ubiquitin ligases targeting Rac1, such as IAPs [19-21]. Ectodermal tissues in mouse embryos might express these proteins to provide robustness to loss of Hace1 protein. Most recently, it has been reported that knockdown of hace1 using a spliceblocking MO causes ROS production in zebrafish [5]. Although this report has not described the effects of the $\mathrm{MO}$ on zebrafish embryonic development, the gross morphology of embryos injected with the MO is apparently normal. Whereas a splice-blocking morpholino inhibits only zygotic expression but not maternal 
expression, a translation blocking morpholino, which was used in our study, inhibits both maternal expression and zygotic expression. This might explain the difference between our results and those from the previous study in zebrafish.

hace1 depletion in Xenopus laevis embryos led to diverse developmental defects, including a shortened body axis and the inhibition of eye and pigment formation. These defects were at least partly rescued by rac1 inhibition. In addition, we showed that overexpression of active Rac1 in the presumptive neural plate led to a delay in neural tube closure and a number of subsequent developmental defects, which are similar to hace1 morphants. The migration of neural crest cells, which give rise to pigment cells, is disturbed by constitutively active or dominant negative forms of Rac1 [22]. Therefore, the inhibition of pigment formation may result from impaired neural crest migration. Additionally, conditional knockout of Racl in the surface ectoderm results in a failure of neural tube closure in mice [23]. Thus, fine tuning of Rac1 activity may be important for neural tube closure. However, the detailed molecular mechanism by which excessive active Rac1 affects neural tube closure is unclear. Rac1 regulates a variety of biological processes, including cytoskeleton remodeling and PCP signaling regulation [24, 25]. PCP signaling plays an important role in neural tube closure [26], and there may be crosstalk between RhoA and Rac1 signaling in the regulation of actin cytoskeleton [27]. It would be interesting to investigate the presence of the crosstalk and, if it is present, to clarify the regulation mechanism of the PCP pathway through RhoA and Rac1 signaling during neural tube closure.

\section{Conclusions}

In summary, our study show that the E3 ubiquitin ligase Hace1 plays an important role in early developmental processes in Xenopus laevis. Detailed analyses of downstream targets of Hace1 should be performed in future studies.

\section{Methods}

\section{Molecular cloning and plasmid construction}

Primers were designed on the basis of the Xenopus laevis hace1 sequence (GenBank accession NM_001093608) as follows: F, 5 '-GGTAGATCTATGGAGAGAGCAATGGAG CAACTC-3'; R, 5'-GGTAGATCTTTATGCCATTGTGT AGCCGTAGCT-3', and the Xenopus laevis rac1 sequence (GenBank accession NM_001095863) as follows: F, 5'- CG GAAGATCTGTAGGGAGAGCAAAGAAGAGGGAGGG AG-3'; R, 5' - TCCGGAATTCAGGGACAGAAGAAAAG ATGGCATGTGGG-3'. PCR was performed with complementary DNAs derived from embryos at stage 2 for hace 1 and stage 13 for racl. The entire amplified coding sequences were cloned into the expression vector pCS2+. A constitutively active form of Xenopus rac1 was constructed by replacing glycine 12 with valine by sitedirected mutagenesis.

\section{Whole mount in situ hybridization}

Whole-mount in situ hybridization was performed on albino Xenopus laevis embryos according to a standard protocol [28] using a robot (InSituPro, Intavis). The digoxigenin-labeled probes were synthesized from hace1pCS2+.

\section{Embryo manipulation}

Xenopus laevis embryos were obtained by in vitro fertilization and cultured in $0.1 \times \mathrm{MBS}$ at 15 or $22{ }^{\circ} \mathrm{C}$. Embryos were staged according to Nieuwkoop and Faber [29]. Antisense morpholino oligonucleotides (MOs) and mRNAs were injected into the animal region of one or two dorsal blastomeres at the four-cell stage in $4 \%$ Ficoll in $0.1 \times$ MBS. In vitro synthesis of capped mRNA was performed using the mMESSAGE mMACHINE (Ambion). MOs were purchased from Gene Tools. The MO sequences were as follows: HACE1 MO, 5' GAGTTGCTCCATTGCTCTCTCCATC-3'; HACE1 MO2, 5' - AGAGGCTCAGCAGTTCCTAAGCAGT-3'; Rac1 MO, 5'-CCACACATTTAATGGCCTGCATGGC$3^{\prime}$; and a standard control oligo (Control MO), 5' CCTCTTACCTCAGTTACAATTTATA-3'. The animals were bred and handled with care, according to a published laboratory manual [28]. Xenopus laevis experiments complied with the Regulation on Animal Experimentation at Kyoto University and were approved by the Animal Experimentation Committee of Kyoto University.

\section{Immunoblotting}

Embryos were lysed in a buffer consisting of $20 \mathrm{mM}$ Tris-HCl, pH 7.5, $150 \mathrm{mM} \mathrm{NaCl}, 1.5 \mathrm{mM} \mathrm{MgCl}_{2}, 2 \mathrm{mM}$ EGTA, $25 \mathrm{mM} \beta$-glycerophosphate, $10 \mathrm{mM}$ sodium pyrophosphate, $1 \%$ Nonidet P-40, $10 \mathrm{mM} \mathrm{NaF}, 1 \mathrm{mM}$ vanadate, $1 \mathrm{mM}$ DTT, and $1 \times$ Protein Inhibitor Cocktail (Sigma). Extracts were then centrifuged, and supernatants were collected. Anti-Myc (A14, Santa Cruz), antiGFP (JL8, Clontech), anti-Rac1 (23A8, Millipore) or anti- $\alpha$-tubulin (DM1A, Sigma) antibodies were used as primary antibodies, and anti-mouse IgG HRPconjugated (1:10,000; GE healthcare) and anti-rabbit IgG HRP-conjugated (1:10,000; GE healthcare) were used as secondary antibodies.

\section{Quantification of body length}

Embryos were fixed in MEMFA [100 mM MOPS (pH 7.4), $2 \mathrm{mM}$ EGTA, $1 \mathrm{mM} \mathrm{MgSO}$, $3.7 \%$ formaldehyde] at stage 40-41. Embryos were placed on agarose gel, and images 
were collected. The body length was measured along the dorsal midline using the AxioVision (Zeiss) measure tool.

\section{Keller sandwich explant analysis}

Keller sandwich explant experiments were performed as previously described [30]. MOs and mRNA were injected into the animal region of two dorsal blastomeres at the four-cell stage. Injected embryos were grown to stage 10.5 for preparation of Keller sandwich explants. Explants were cultured in Sater's modified blastocoel buffer containing $0.1 \%$ BSA until uninjected embryos reached stage 19 . The length and width of explants were measured using cellSens software (Olympus).

\section{Time-lapse analysis of neural tube closure}

Embryos injected with MOs or mRNAs were grown to stage 13 and placed dorsal side up in a $60 \mathrm{~mm}$ culture dish. This dish contained $2 \%$ agarose with a groove made by an acrylic comb. Images were collected with an Olympus SZX16 microscope, and time-lapse movies were obtained using cellSens software. For quantitative analysis of the delay in neural tube closure, the maximum distance between the dorsal midline and the left (i) or right (ii) inner edge of the neural fold was measured at the time point $60 \mathrm{~min}$ after start of time-lapse imaging, as shown in Fig. 4c. At the time point 60 min after start of time-lapse imaging, embryos reached stage 17. The inner edge of the neural fold was easily visible, owing to pigmentation.

\section{Quantitative RT-PCR analysis}

Total RNA isolation and cDNA synthesis were performed as previously described [31]. The gene expression levels were normalized to those of odc1 (ornithine decarboxylase 1). The sequences of primers used were as follows: Xenopus laevis rac1 (NM_001095863) (F, CATGCACATGTCA AGCCAGTTC; R, ATGGCAAGTCCCTGAGGATAGG); Xenopus laevis rac2 (NM_001092288) (F, ACCAGTA AACTTGGGCTTGTGG; R, CTCATAAGATGCCGGA CTCACC); Xenopus laevis odc1 (NM_001086698) (F, TGAAAGTGGCAAGGAATCACCC; R, GATACGAT CCAGCCCATCACAC).

\section{Additional files}

Additional file 1: Movie S1. Neural tube closure of an uninjected embryo. (MOV $157 \mathrm{~kb}$ )

Additional file 2: Movie S2. Neural tube closure of an embryo injected with HACE1 MO. (MOV 190 kb)

Additional file 3: Movie S3. Neural tube closure of an embryo injected with Rac1-V12 mRNA. (MOV 86.9 kb)

\section{Abbreviations}

GAP: GTPase-activating protein; HACE1: HECT domain and ankyrin repeat containing E3 ubiquitin protein ligase 1; IAP: Inhibitors of apoptosis protein; MO: Morpholino oligonucleotides; ROS: Reactive oxygen species
Acknowledgments

We thank members of the Nishida laboratory for technical advice and helpful discussions.

\section{Funding}

This work was supported by a grant from the Ministry of Education, Culture, Sports, Science and Technology of Japan (to MK) and the Takeda Science Foundation (to MK)

Availability of data and materials

All supporting data are presented in the main manuscript and supplementary data files.

\section{Authors' contributions}

$\mathrm{Al}, \mathrm{FY}, \mathrm{TS}$, and TE designed experiments. Al, FY, and TE performed experiments. Al, FY, TS, EN, and MK wrote the manuscript. All authors read and approved the final manuscript.

\section{Competing interests}

The authors declare that they have no competing interests.

\section{Consent for publication}

Not applicable.

\section{Ethics approval and consent to participate}

Xenopus laevis experiments complied with the Regulation on Animal Experimentation at Kyoto University and were approved by the Animal Experimentation Committee of Kyoto University.

Received: 12 November 2015 Accepted: 25 August 2016

Published online: 21 September 2016

\section{References}

1. Anglesio MS, Evdokimova V, Melnyk N, Zhang L, Fernandez CV, Grundy PE, et al. Differential expression of a novel ankyrin containing E3 ubiquitinprotein ligase, Hace1, in sporadic Wilms' tumor versus normal kidney. Hum Mol Genet. 2004;13(18):2061-74.

2. Zhang L, Anglesio MS, O'Sullivan M, Zhang F, Yang G, Sarao R, et al. The E3 ligase HACE1 is a critical chromosome 6q21 tumor suppressor involved in multiple cancers. Nat Med. 2007;13(9):1060-9.

3. Torrino S, Visvikis $O$, Doye A, Boyer L, Stefani C, Munro P, et al. The E3 ubiquitin-ligase HACE1 catalyzes the ubiquitylation of active Rac1. Dev Cell. 2011;21(5):959-65.

4. Castillo-Lluva S, Tan CT, Daugaard M, Sorensen PH, Malliri A. The tumour suppressor HACE1 controls cell migration by regulating Rac1 degradation Oncogene. 2013;32(13):1735-42.

5. Daugaard M, Nitsch R, Razaghi B, McDonald L, Jarrar A, Torrino S, et al. Hace1 controls ROS generation of vertebrate Rac1-dependent NADPH oxidase complexes. Nat Commun. 2013:4:2180.

6. Tang D, Xiang Y, De Renzis S, Rink J, Zheng G, Zerial M, et al. The ubiquitin ligase HACE1 regulates Golgi membrane dynamics during the cell cycle. Nat Commun. 2011;2:501.

7. Lachance V, Degrandmaison J, Marois S, Robitaille M, Genier S, Nadeau S, et al. Ubiquitylation and activation of a Rab GTPase is promoted by a B2AR-HACE1 complex. J Cell Sci. 2014;127(Pt 1):111-23.

8. Rotblat B, Southwell AL, Ehrnhoefer DE, Skotte NH, Metzler M, Franciosi S, et al. HACE1 reduces oxidative stress and mutant Huntingtin toxicity by promoting the NRF2 response. Proc Natl Acad Sci U S A. 2014:111(8):3032-37.

9. Zhang L, Chen X, Sharma P, Moon M, Sheftel AD, Dawood F, et al. HACE1-dependent protein degradation provides cardiac protection in response to haemodynamic stress. Nat Commun. 2014;5:3430.

10. Keller R. Shaping the vertebrate body plan by polarized embryonic cell movements. Science. 2002;298(5600):1950-54.

11. Keller $\mathrm{R}$, Danilchik M. Regional expression, pattern and timing of convergence and extension during gastrulation of Xenopus laevis. Development. 1988;103(1):193-209.

12. Eisen JS, Smith JC. Controlling morpholino experiments: don't stop making antisense. Development. 2008;135(10):1735-43.

13 Lucas JM, Nikolic I, Hens MD. cDNA cloning, sequence comparison, and developmental expression of Xenopus rac1. Mech Dev. 2002;115(1-2):113-6. 
14 Tahinci E, Symes K. Distinct functions of Rho and Rac are required for convergent extension during Xenopus gastrulation. Dev Biol. 2003;259(2):318-35.

15 Magdaleno S, Jensen P, Brumwell CL, Seal A, Lehman K, Asbury A, et al. BGEM: an in situ hybridization database of gene expression in the embryonic and adult mouse nervous system. PLoS Biol. 2006;4(4):e86.

16 Davidson LA, Keller RE. Neural tube closure in Xenopus laevis involves medial migration, directed protrusive activity, cell intercalation and convergent extension. Development. 1999;126(20):4547-56.

17 Wallingford JB, Harland RM. Neural tube closure requires Dishevelleddependent convergent extension of the midline. Development. 2002;129(24):5815-25.

18 Wallingford JB, Harland RM. Xenopus Dishevelled signaling regulates both neural and mesodermal convergent extension: parallel forces elongating the body axis. Development. 2001;128(13):2581-92.

19 Nethe M, Hordijk PL. The role of ubiquitylation and degradation in RhoGTPase signalling. J Cell Sci. 2010;123(Pt 23):4011-18.

20 Oberoi TK, Dogan T, Hocking JC, Scholz RP, Mooz J, Anderson CL, et al. IAPs regulate the plasticity of cell migration by directly targeting Rac1 for degradation. EMBO J. 2012;31(1):14-28.

21 Oberoi-Khanuja TK, Rajalingam K. IAPs as E3 ligases of Rac1: shaping the move. Small GTPases. 2012;3(2):131-36.

22 Shoval I, Kalcheim C. Antagonistic activities of Rho and Rac GTPases underlie the transition from neural crest delamination to migration. Dev Dyn. 2012;241(7):1155-68.

23 Camerer E, Barker A, Duong DN, Ganesan R, Kataoka H, Cornelissen I, et al. Local protease signaling contributes to neural tube closure in the mouse embryo. Dev Cell. 2010;18(1):25-38.

24 Bosco EE, Mulloy JC, Zheng Y. Rac1 GTPase: a "Rac" of all trades. Cell Mol Life Sci. 2009;66(3):370-4.

25 Gao C, Chen YG. Dishevelled: The hub of Wnt signaling. Cell Signal. 2010;22(5):717-27.

26 Wallingford JB. Planar cell polarity, ciliogenesis and neural tube defects. Hum Mol Genet. 2006;15(Spec No 2):R227-34.

27 Lessey EC, Guilluy C, Burridge K. From mechanical force to RhoA activation. Biochemistry. 2012:51(38):7420-32.

28 Sive HL, Grainger RM, Harland RM. Early development of Xenopus laevis: a laboratory manual. New York: Cold Spring Harbor Laboratory Press; 2000.

29 Nieuwkoop PD, Faber J. Normal table of Xenopus laevis (daudin). Amersterdam: North Holland Publishing Company; 1967.

30 Sive HL, Grainger RM, Harland RM: Xenopus laevis Keller Explants. CSH protocols. 2007; doi:10.1101/pdb.prot4749.

31 Suzuki T, Kusakabe M, Nakayama K, Nishida E. The protein kinase MLTK regulates chondrogenesis by inducing the transcription factor Sox6. Development. 2012:139(16):2988-98.

\section{Submit your next manuscript to BioMed Central and we will help you at every step:}

- We accept pre-submission inquiries

- Our selector tool helps you to find the most relevant journal

- We provide round the clock customer support

- Convenient online submission

- Thorough peer review

- Inclusion in PubMed and all major indexing services

- Maximum visibility for your research

Submit your manuscript at www.biomedcentral.com/submit
Biomed Central 